\title{
Avaliação terapêutica e posológica da levotiroxina sódica em cães com hipotiroidismo primário adquirido ${ }^{1}$
}

\author{
Viviani De Marco ${ }^{2 *}$, Roberta M.T. Silva ${ }^{3}$, Mariana A. Karamm ${ }^{4}$, Jorge C. Florio ${ }^{5}$ \\ e Carla A.B. Lorigrados ${ }^{6}$
}

\begin{abstract}
De Marco V., Silva R.M.T., Karamm M.A., Florio J.C. \& Lorigrados C.A.B. 2012. [L-levothyroxine therapeutic dosage evaluation in dogs with primary hypothyroidism.] Avaliação terapêutica e posológica da levotiroxina sódica em cães com hipotiroidismo primário adquirido. Pesquisa Veterinária Brasileira 32(10):1030-1036. Setor de Clínica Médica, Hospital Veterinário, Faculdade de Veterinária, Universidade Santo Amaro, Rua José Portolano 57, Jardim das Imbuias, São Paulo, SP 04829-300, Brazil. E-mail: vivianidemarco@terra.com.br

The acquired primary hypothyroidism is a frequently diagnosed endocrinopathy in dogs. The therapy constitutes in oral supplementation with sodium levothyroxine (L-thyroxine), however various therapeutic protocols have been proposed in the literature, with doses ranging from 11 to $44 \mathrm{mg} / \mathrm{kg}$ once or twice a day, since L-thyroxine has a great variability of absorption and plasma half life. We studied 30 dogs with primary hypothyroidism (13 males and 17 females, mean age $7.9 \pm 1.9$ years and mean weight of $19.1 \pm 12.6 \mathrm{~kg}$ ), in order to evaluate the dose and frequency of administration of L-thyroxine used more often able to secure a satisfactory therapeutic control as measured by clinical signs and test post-pill, and to correlate the amount of thyroxine employed with the animals' weight. The mean dose of thyroxine used in our study was $16.9 \pm 3.1 \mathrm{mg} / \mathrm{kg}$, and the frequency of administration every 12 hours in $50 \%$ of cases. To investigate a possible correlation between weight and dose of thyroxine used, since small dogs have a higher metabolic rate than large dogs, the animals were grouped in Group A, dogs weighing $\leq 10 \mathrm{~kg}(\mathrm{n}=12 / 30,7.7 \pm 2.1 \mathrm{~kg})$ and group B, dogs weighing $>10 \mathrm{~kg}(\mathrm{n}=18 / 30,26.8 \pm 10.7 \mathrm{~kg})$. The mean dose of thyroxine used in groups $A$ and B did not differ significantly and were respectively $16 \pm 3 \mathrm{mg} / \mathrm{kg}$ and $17 \pm 3 \mathrm{mg} / \mathrm{kg}$. The frequency of administration was 50\% every 24 hours and 50\% every 12 hours for both groups. Thus, the dose of thyroxine does not seem to correlate with the weight of the animal being unpredictable who should receive the highest dose and frequency of the medication. The protocol should be individualized and the patient adequately monitored.
\end{abstract}

INDEX TERMS: Primary hypothyroidism, L-thyroxine, therapy, dogs.

\footnotetext{
${ }^{1}$ Recebido em 24 de fevereiro de 2012.

Aceito para publicação em 24 de abril de 2012.

2 Docente, Faculdade de Medicina Veterinária, Universidade Santo Amaro (Unisa), Rua Bernardo dos Santos 10, Apto 162, Beta, Jardim Olympia, São Paulo, SP 05542-000, Brasil. *Autor para correspondência: vivianidemarco@terra.com.br

${ }^{3}$ Hospital Veterinário da Universidade Guarulhos (UNG), Rua Brasilianas 56, São Miguel, SP 08020-280, Brasil. E-mail: bertamt@hotmail.com

${ }^{4}$ Hospital Veterinário Pompéia, Rua Iperoig 899, Apto 33, Perdizes, São Paulo, SP 05016-000. E-mail: marikaramm@gmail.com

5 Docente, Faculdade de Medicina Veterinária, Universidade de São Paulo (USP), Av. Diogenes Ribeiro de Lima 2170, Apto 131, Alto de Pinheiros, São Paulo, SP 05458-001. E-mail: jflorio@usp.br

6 Docente, Faculdade de Medicina Veterinária, Faculdades Metropolitanas Unidas (FMU), Rua Felix Guilherme 846, Lapa, São Paulo, SP 05069000. E-mail: wcpf@ig.com.br
}

RESUMO.- 0 hipotireoidismo primário adquirido é uma endocrinopatia frequentemente diagnosticada na espécie canina. A terapia consiste na suplementação oral com levotiroxina sódica (L-tiroxina), no entanto vários protocolos terapêuticos têm sido propostos pela literatura, com doses variando 11 a $44 \mu \mathrm{g} / \mathrm{kg}$ uma a duas vezes ao dia, visto à grande variabilidade de absorção e meia-vida plasmática do fármaco. Foram estudados 30 cães com hipotiroidismo primário adquirido (13 machos e 17 fêmeas, idade média de 7,9 $\pm 1,9$ anos e peso médio de $19,1 \pm 12,6 \mathrm{~kg}$ ) atendidos no Hospital Veterinário da Universidade Guarulhos (UnG) e no Serviço de Endocrinologia de duas clínicas particulares da cidade de São Paulo (2009-2011), com o objetivo de avaliar a posologia e a frequência de administração da L- 
-tiroxina, mais frequentemente utilizada, capaz de garantir um controle terapêutico satisfatório, avaliado através dos sinais clínicos e do teste pós-tiroxina, além de correlacionar a dose de tiroxina empregada com o peso dos animais. A dose média de tiroxina utilizada em nossa casuística foi de $16,9 \pm 3,1 \mu \mathrm{g} / \mathrm{kg}$, sendo a frequência de administração a cada 12 horas em 50\% dos casos. Para se investigar uma possível correlação entre o peso e a dosagem de tiroxina utilizada, uma vez que cães de pequeno porte apresentam maior taxa metabólica que cães de grande porte, os animais foram agrupados em grupo $A$, cães com peso $\leq 10 \mathrm{Kg}$ $(\mathrm{n}=12 / 30 ; 7,7 \pm 2,1 \mathrm{~kg})$ e grupo $\mathrm{B}$, cães com peso $>10 \mathrm{~kg}$ $(\mathrm{n}=18 / 30,26,8 \pm 10,7 \mathrm{~kg})$. A dose média de tiroxina empregada nos grupos A e B não apresentaram diferença estatística e foram, respectivamente, $16 \pm 3 \mu \mathrm{g} / \mathrm{kg}$ e $17 \pm 3 \mu \mathrm{g} / \mathrm{kg}$. A frequência de administração foi $50 \%$ a cada 24 horas e $50 \%$ a cada 12 horas para ambos os grupos. Dessa forma, a dose de tiroxina não parece se correlacionar com o peso do animal, sendo imprevisível quem deverá receber dose e frequência máxima da medicação. 0 protocolo deve ser individualizado e o paciente devidamente monitorado.

TERMOS DE INDEXAÇÃO: Hipotiroidismo primário, levotiroxina, tratamento, cães.

\section{INTRODUÇÃO}

O hipotiroidismo é uma doença endócrina frequentemente diagnosticada na espécie canina, caracterizado pela hipofunção da glândula tiróide resultando em produção deficiente dos hormônios tiroideanos (Feldman \& Nelson 2004). 0 hipotiroidismo primário é responsável por $95 \%$ dos casos e tem como causa uma tiroidite linfocítica auto-imune ou uma atrofia idiopática das glândulas tiróides (Nelson \& Elliott 2006, Graham et al. 2007).

A avaliação da função da glândula tiróide em cães suspeitos de hipotiroidismo é feita através da mensuração sérica de T4 (tiroxina), tanto ligado às proteínas (T4 total -T4t), como na sua forma livre (T4 livre - T4l), além da concentração sérica de TSH (tireotropina). A constatação de níveis baixos de T4t e T4l e níveis altos de TSH em um cão com história clínica sugestiva de hipofunção tiroideana suportam um diagnóstico de hipotiroidismo primário (Nelson \& Elliott 2006). A mensuração da concentração do hormônio estimulante da tiróide (TSH) é útil no diagnóstico de cães com suspeita de hipotireoidismo, porém deve ser interpretada com cautela, devido a sua baixa sensibilidade. Por este motivo, níveis de TSH normais não devem excluir o hipotiroidismo (Peterson et al. 1997).

O tratamento de eleição para o hipotiroidismo primário é a administração oral de hormônio tiroideano sintético, a levotiroxina sódica (L-tiroxina), que resulta em aumento dos níveis plasmáticos de tiroxina (T4), aumento de triiodotironina (T3), uma vez que esses produtos podem ser convertidos à forma metabolicamente ativa T3 pelos tecidos periféricos, e redução dos níveis de tireotropina (TSH) pela retroalimentação negativa sobre a hipófise (Feldman \& Nelson 2004).

O objetivo da suplementação com L-tiroxina é determinar a dose ideal capaz de controlar os sinais clínicos sem induzir uma tireotoxicose. A dose geralmente instituída é de 20 a $22 \mu \mathrm{g} / \mathrm{kg}$ a cada 12 horas (Scott-Moncrieff 2010), no entanto diferentes protocolos terapêuticos têm sido recomendados, com doses variando amplamente de 11 a $44 \mu \mathrm{g} /$ kg uma a duas vezes ao dia (Traon et al. 2010). Tal variação se deve em parte à grande variabilidade de absorção do fármaco, com biodisponibilidade oral estimada em torno de 10 a $50 \%$ apenas, e a curta meia vida plasmática do fármaco, estimada em 10 a 14 horas na espécie canina. A administração do fármaco junto com alimentos reduz ainda mais sua absorção, devendo o animal ser submetido a jejum alimentar pelo menos uma a duas horas antes e após a medicação. Além disso, existem diferenças de potência e de biodisponibilidade entre os diferentes produtos comerciais a base de L-tiroxina, sendo os produtos veterinários considerados superiores aos produtos humanos (Scott-Moncrieff 2010).

A melhor forma de monitorar o tratamento do hipotiroidismo é através da realização do teste pós tiroxina, no qual se avalia a concentração de T4t basal e T4t 6 horas após a administração da tiroxina exógena. 0 T4t deve apresentar valores dentro da normalidade na amostra basal $(1,5-4,0 \mu \mathrm{g} / \mathrm{dL})$ e valores no limite superior da normalidade ou discretamente elevados na amostra 6 h pós tiroxina $(2,5-$ $5,0 \mu \mathrm{g} / \mathrm{dL}$ ). Valores de T4t pós-tiroxina entre 5,0 e 6,0 $\mu \mathrm{g} / \mathrm{dL}$ em cães clinicamente bem e assintomáticos podem ser considerados normais. Esta forma de avaliação é clinicamente eficaz, mas pode permitir um excesso de suplementação hormonal em alguns animais (Scott-Moncrieff 2010). Qualquer valor inferior a 1,5 $\mu \mathrm{g} / \mathrm{dL}$ sugere controle terapêutico inadequado, implicando em necessidade de reajuste de dose ou aumento da frequência de administração do fármaco. Caso o regime de administração seja inicialmente a cada 12 horas, pode-se interpretar somente o T4t pós tiroxina, obedecendo os mesmos valores de referência de 2,5 a 5,0 $\mu \mathrm{g} / \mathrm{dL}$ (De Marco 2008).

Associado ao monitoramento hormonal é imprescindível o monitoramento clínico com observação de melhora da letargia e aumento da atividade física já nas primeiras duas semanas e perda de peso evidente após quatro a oito semanas. Além disso, é conveniente avaliar a normalização dos níveis de colesterol e triglicérides. Sugere-se a realização do teste pós tiroxina 4 e 8 semanas após o início da terapia e, posteriormente, a cada 3 meses, devido à variação individual de resposta ao tratamento. Alterações na posologia são necessárias em aproximadamente $50 \%$ dos casos ao longo do tratamento (Dixon et al. 2002, Scott-Moncrieff 2010).

A expectativa de vida de um cão com hipotiroidismo primário, submetido à suplementação hormonal adequada deve ser igual a de cães hígidos. A maioria das manifestações clínicas, se não todas, serão resolvidas com o tratamento (Nelson \& Elliott 2006).

Devido à existência de variabilidade na absorção e metabolismo da droga, bem como os diferentes estágios da doença que pode conferir alguma função residual da glândula, a dose e a frequência da tiroxina exógena devem ser analisadas individualmente a cada paciente, a fim de se obter sucesso terapêutico sem causar efeitos colaterais. 
Observa-se, na prática clínica, que nem todos os cães com hipotiroidismo primário necessitam da dose de $20 \mu \mathrm{g} / \mathrm{kg}$ a cada $12 \mathrm{~h}$ de L-tiroxina, por via oral, pois notável melhora clínica pode ser observada em animais submetidos a doses inferiores ou frequência a cada 24 horas. Embora, acredita-se que cães sejam mais resistentes ao desenvolvimento de hipertiroidismo iatrogênico, devido a curta meia vida plasmática do fármaco, a tireotoxicose pode se desenvolver em cães suplementados com doses excessivas de L-tiroxina e/ou tratados duas vezes ao dia (Scott-Moncrieff 2010). Utilizando-se a menor dose possível do fármaco capaz de garantir um bom controle clínico e hormonal, podem-se minimizar os custos e facilitar a adesão do proprietário ao tratamento, quando o mesmo for administrado a cada 24 horas.

Sendo assim, o presente estudo teve como objetivos avaliar a posologia e a frequência de administração da levotiroxina sódica mais frequentemente utilizada para tratar satisfatoriamente cães com hipotiroidismo primário adquirido, e avaliar existe alguma correlação entre os diferentes protocolos terapêuticos com o peso dos animais.

\section{MATERIAL E MÉTODOS}

Nossa casuística foi composta de 30 cães (13 machos e 17 fêmeas) com diagnóstico de hipotiroidismo primário adquirido, sendo
11 animais com raça definida e 19 sem raça definida, com idade média de $7,9 \pm 1,9$ anos e peso médio de $19,1 \pm 12,6 \mathrm{~kg}$ (variando de 4 a $45 \mathrm{~kg}$ ), atendidos no serviço de Clínica Médica do Hospital Veterinário da Faculdade de Medicina Veterinária da Universidade Guarulhos (UnG) e no Serviço de Endocrinologia de duas clínicas particulares da cidade de São Paulo (Hospital Veterinário Pompéia e NAYA Especialidades) no período de 2009 a 2011. Dentre os animais com raça definida, destacaram-se as raças: Maltês $(n=4 / 30 ; 13,6 \%)$, Poodle $(n=4 / 30 ; 13,6 \%)$, Golden Retriever $(n=3 / 30,10 \%)$, Labrador $(n=3 / 30,10 \%)$, Cocker $(n=2 / 30,6,6 \%)$, Pit Bull ( $n=2 / 30 ; 6,6 \%)$, Schnauzer miniatura $(n=1 / 30 ; 3,3 \%)$, Scottish Terrier ( $\mathrm{n}=1 / 30 ; 3,3 \%)$ e Pastor de Shetland $(n=1 / 30$; $3,3 \%)$.

O diagnóstico foi embasado nos dados de anamnese e exame físico, buscando alterações clínicas típicas de hipotiroidismo como letargia, ganho de peso, termofilia, alopecia simétrica e bilateral, "face trágica", "cauda de rato", piodermite recidivante; pesquisa de hiperlipidemia, através da dosagem sérica de triglicérides (Método Ponto Final; kit Labtest) e colesterol (Método Ponto Final; kit Labtest), além da realização dos testes hormonais que avaliam a função tiroideana, tais como T4 total (pelo método de radioimunoensaio), T4 livre (pelo método de diálise bifásica) e TSH canino (pelo método de radioimunoensaio). As dosagens hormonais foram encaminhadas a um Laboratório Veterinário Especializado da cidade de São Paulo ${ }^{7}$, que utilizou kits humanos Coat-A-Count $®$ da

\footnotetext{
${ }^{7}$ Provet ${ }^{\circledR}$ Medicina Veterinária Diagnóstica, Avenida Aratãs 1009, Moema, SP.
}

Quadro 1. Idade, sexo, raça e valores de exames laboratoriais empregados para o diagnóstico de 30 cães com hipotiroidismo primário (SP, 2009-2011)

\begin{tabular}{ccccccccc}
\hline Animal & $\begin{array}{c}\text { Sexo } \\
\text { (M/F) }\end{array}$ & Raça & $\begin{array}{c}\text { Idade } \\
\text { (anos) }\end{array}$ & $\begin{array}{c}\text { T4 total } \\
(\mu \mathrm{g} / \mathrm{dL})\end{array}$ & $\begin{array}{c}\text { T4 livre } \\
(\mathrm{ng} / \mathrm{dL})\end{array}$ & $\begin{array}{c}\text { TSH } \\
(\mathrm{ng} / \mathrm{mL})\end{array}$ & $\begin{array}{c}\text { Colesterol } \\
(\mathrm{mg} / \mathrm{dL})\end{array}$ & $\begin{array}{c}\text { Triglicérides } \\
(\mathrm{mg} / \mathrm{dL})\end{array}$ \\
\hline 1 & $\mathrm{M}$ & SRD & 13 & $*$ & 0,1 & 0,4 & 233 & 87 \\
2 & $\mathrm{M}$ & SRD & 12 & $*$ & 0,1 & $*$ & 238 & $*$ \\
3 & $\mathrm{M}$ & SRD & 8 & $*$ & 0,17 & $*$ & 569 & $*$ \\
4 & $\mathrm{~F}$ & Cocker Spaniel & 9 & 1,0 & 0,1 & $*$ & 150 & 61 \\
5 & $\mathrm{~F}$ & SRD & 11 & 0,54 & 0,3 & $*$ & 557 & 135 \\
6 & $\mathrm{~F}$ & SRD & 11 & 0,5 & 0,02 & $*$ & 322 & 87 \\
7 & $\mathrm{~F}$ & Poodle & 7 & 0,7 & 0,4 & 0,6 & 320 & 140 \\
8 & $\mathrm{~F}$ & Golden Retriever & 8 & 0,3 & 0,03 & 0,8 & 345 & 167 \\
9 & $\mathrm{~F}$ & Scottish terrier & 8 & 0,34 & 0,2 & 0,7 & 280 & 90 \\
10 & $\mathrm{M}$ & Maltês & 7 & 0,6 & 0,25 & 0,55 & 250 & 85 \\
11 & $\mathrm{M}$ & Maltês & 5 & 0,7 & 0,1 & $*$ & 239 & 108 \\
12 & $\mathrm{~F}$ & Labrador & 8 & 0,4 & 0,47 & 0,61 & 248 & 97 \\
13 & $\mathrm{M}$ & SRD & 8 & 1,0 & 0,1 & $*$ & 258 & 62 \\
14 & $\mathrm{~F}$ & Cocker Spaniel & 6 & $*$ & 0,45 & 0,4 & $*$ & $*$ \\
15 & $\mathrm{M}$ & Maltês & 8 & 0,37 & $*$ & 0,72 & 1850 & 439 \\
16 & $\mathrm{~F}$ & SRD & 7 & 0,41 & 0,01 & $*$ & 371 & 121 \\
17 & $\mathrm{~F}$ & Pit Bull & 5 & 0,36 & 0,46 & 1,2 & 207 & 40 \\
18 & $\mathrm{~F}$ & Poodle & 7 & 0,29 & 0,01 & & 662 & 286 \\
19 & $\mathrm{~F}$ & Poodle & 13 & 0,4 & 0,32 & 1,82 & 302 & 101 \\
20 & $\mathrm{~F}$ & Pastor de Shetland & 6 & 0,3 & 0,14 & 0,37 & 813 & 116 \\
21 & $\mathrm{M}$ & Golden Retriever & 5 & $*$ & 0,35 & 0,7 & 562 & 768 \\
22 & $\mathrm{~F}$ & Labrador & 8 & $*$ & 0,22 & 0,45 & 632 & 350 \\
23 & $\mathrm{M}$ & SRD & 9 & 0,5 & 0,43 & 0,6 & 448 & 507 \\
24 & $\mathrm{M}$ & Labrador & 9 & 0,6 & 0,29 & 1,25 & 425 & 82 \\
25 & $\mathrm{~F}$ & Poodle & 10 & $*$ & 0,01 & 0,75 & 420 & $*$ \\
26 & $\mathrm{M}$ & SRD & 7 & 0,5 & 0,27 & 0,45 & 193 & 350 \\
27 & $\mathrm{M}$ & Golden Retriever & 7 & 0,6 & 0,3 & 0,6 & 350 & 280 \\
28 & $\mathrm{~F}$ & Maltês & 6 & 0,3 & 0,19 & 1,25 & 473 & 785 \\
29 & $\mathrm{M}$ & Schnauzer & 9 & 0,4 & 0,25 & 0,75 & 450 & 850 \\
30 & $\mathrm{~F}$ & Pit Bull & 6 & 0,19 & 0,21 & 0,45 & 435 & 250 \\
Média & & & 7,93 & 0,49 & 0,21 & 0,73 & 434 & 247 \\
Desvio padrão & & 1,99 & 0,21 & 0,14 & 0,36 & 315 & 238 \\
VR & & & $1,5-4,0$ & $0,7-2,0$ & $0,1-0,5$ & $135-270$ & $50-100$ \\
& & & & & & &
\end{tabular}

$\mathrm{VR}=$ valor de referencia; ${ }^{*}$ exames não realizados. 
Siemens Medical Solutions Diagnostics, com devida curva de validação para a espécie canina, para análise de T4 total e o kit Gamma Coat TM Free T4 (Two-step) da Dia Sorin para a análise de T4 livre pelo método de extração bifásico. Para análise do TSH canino foi utilizado um kit específico para esta espécie da Coat-A-Count@ Canine TSH IRMA da Siemens Medical Solutions Diagnostics. O protocolo utilizado foi o recomendado pelo fabricante e os padrões de qualidade foram empregados de acordo com o utilizado na rotina do laboratório Provet ${ }^{\circledR}$. Os dados referentes à idade, sexo, raça e exames laboratoriais empregados para o diagnóstico de hipotiroidismo em 30 cães estão descritos no Quadro 1.

Como critérios de inclusão, além de sintomatologia clínica sugestiva de hipotiroidismo, os animais deveriam apresentar obrigatoriamente níveis baixos de T4 livre $(<0,7 \mathrm{ng} / \mathrm{dL})$, exame este considerado o mais específico para o diagnóstico desta enfermidade (Dixon 2009). Os critérios de exclusão compreenderam: presença de outras endocrinopatias ou doenças sistêmicas graves concomitantes e terapia com glicocorticóides, sulfas, fenobarbital, antiinflamatórios não esteroidais, furosemida nos últimos dois meses, a fim de se evitar a presença da síndrome do eutiroideo doente, o que dificultaria a interpretação das dosagens hormonais (Daminet \& Ferguson 2003).

A terapia instituída consistiu de administração oral de levotiroxina sódica humana (Synthroid ${ }^{\circledR}, \mathrm{ABBOTT}$ ), visto que em nosso país, infelizmente, não dispomos de tiroxina veterinária, na dose inicial de $10 \mu \mathrm{g} / \mathrm{kg}$ a cada 24 horas (SID) na primeira semana e, posteriormente, $15 \mu \mathrm{g} / \mathrm{kg}$ a cada 24 horas (SID), sempre pelas manhãs em jejum com oferta de alimentos apenas após $2 \mathrm{~h}$ para otimizar a absorção gastroentérica do fármaco. Na quarta sema-

\section{Quadro 2. Resultados do teste pós-tiroxina (T4t basal e 6 horas após administração de L-tiroxina) de 30 cães com hipotiroidismo primário tratados com levotiroxina sódica na dose de $15 \mu \mathrm{g} / \mathrm{kg} / \mathrm{SID}$ durante 30 dias (SP, 2009-11)}

\begin{tabular}{ccc}
\hline Animal & T4t basal $(\mu \mathrm{g} / \mathrm{dL})$ & T4t 6h pós tiroxina $(\mu \mathrm{g} / \mathrm{dL})$ \\
\hline 1 & 3,8 & 6,2 \\
2 & 1,2 & 2,8 \\
3 & 1,3 & 3,0 \\
4 & 1,2 & 2,5 \\
5 & 1,1 & 2,0 \\
6 & 0,9 & 1,3 \\
7 & 1,9 & 2,4 \\
8 & 1,6 & 2,0 \\
9 & 2,0 & 6,7 \\
10 & 1,7 & 4,8 \\
11 & 1,3 & 2,6 \\
12 & 1,7 & 3,4 \\
13 & 1,9 & 3,3 \\
14 & 1,7 & 2,3 \\
15 & 1,3 & 3,0 \\
16 & 1,6 & 2,4 \\
17 & 2,0 & 7,0 \\
18 & 2,8 & 4,0 \\
19 & 3,5 & 5,0 \\
20 & 2,8 & 4,8 \\
21 & 0,8 & 1,2 \\
22 & 1,0 & 2,0 \\
23 & 1,2 & 2,0 \\
24 & 0,7 & 0,9 \\
25 & 1,1 & 1,8 \\
26 & 1,2 & 2,0 \\
27 & 1,0 & 2,3 \\
28 & 1,5 & 2,4 \\
29 & 1,0 & 2,0 \\
30 & 1,6 & 2,2 \\
Média & 3,0 \\
Desvio padrão & 1,61 & 1,6 \\
& 0,75 &
\end{tabular}

na, foi realizado um retorno com o animal e seu proprietário para avaliação clínica geral e realização do teste pós tiroxina (Quadro 2). Esse teste tem como objetivo avaliar se a reposição hormonal está adequada ou não através das mensurações de T4 total antes (T4t basal) e 6 horas após a administração da tiroxina (T4t pós-tiroxina). Valores de T4t basal entre 1,5 e 4,0 $\mu \mathrm{g} / \mathrm{dL}$ e T4t pós-tiroxina entre 2,5 e 5,0 $\mu \mathrm{g} / \mathrm{dL}$ foram considerados normais. Dessa forma, cães com T4t basal ou T4t pós tiroxina inferiores aos valores de referência citados acima, tiveram sua dose de L-tiroxina ou frequência de administração aumentada. Por outro lado, cães com valores de T4t pós tiroxina superiores a 5,0 $\mu \mathrm{g} / \mathrm{dL}$, tiveram sua dose reduzida. Cães com resultados de teste pós tiroxina dentro da normalidade foram mantidos no tratamento com tiroxina na dose de $15 \mu \mathrm{g} / \mathrm{kg}$ a cada 24 horas (Quadro 3). Após as adequações na dose e frequência da tiroxina, um novo teste pós-tiroxina foi realizado para verificar a normalização das concentrações de T4t (Quadro 4).

Como as doses e frequência de administração da tiroxina são bastante variáveis no tratamento do hipotireoidismo, os animais foram divididos em dois grupos de acordo com seu peso corpóreo, com o intuito de se investigar uma correlação entre a dose do fármaco e o peso do animal. 0 grupo A foi composto de 12 cães com peso inferior ou igual a $10 \mathrm{~kg}(7,7 \pm 2,1 \mathrm{~kg})$ e o grupo B de 18 cães com peso superior a $10 \mathrm{~kg}(17,0 \pm 3,0 \mathrm{~kg}$ ) (Quadro 5).

Para as análises estatísticas foi utilizado o programa estatístico computadorizado GraphPad Instat (versão 3.0). Devido à distribuição gaussiana dos valores numéricos provenientes dos dois grupos de animais (A e B), os pesos e as doses de tiroxina foram analisadas utilizando o teste-t student para dados não pareado. A correlação linear entre o peso e a dose de tiroxina foi investigada através da correlação de Pearson. Valores de $p<0,05$ foram considerados estatisticamente significantes. Além disso, foi realizada a análise estatística descritiva de todas as variáveis (média e desvio padrão).

Quadro 3. Conduta terapêutica de acordo com os resultados do teste pós-tiroxina em cães hipotiroideos tratados com levotiroxina sódica na dose de 15 g/Kg/SID (SP, 2009-2011)

\begin{tabular}{ccc}
\hline $\begin{array}{c}\text { T4 t basal } \\
(\mu \mathrm{g} / \mathrm{dL})\end{array}$ & $\begin{array}{c}\text { T4 t } 6 \text { horas pós- } \\
\text { tiroxina }(\mu \mathrm{g} / \mathrm{dL})\end{array}$ & Conduta terapêutica \\
\hline $1,5-4,0$ & $2,5-5,0$ & Manter a dose $(15 \mu \mathrm{g} / \mathrm{Kg} / \mathrm{SID})$ \\
$<1,5$ & $2,5-5,0$ & Aumentar frequência $(15 \mu \mathrm{g} / \mathrm{Kg} / \mathrm{BID})$ \\
$1,5-4,0$ & $<2,5$ & Aumentar dose $(16$ a $20 \mu \mathrm{g} / \mathrm{Kg} / \mathrm{SID})$ \\
$<1,5$ & $<2,5$ & Aumentar dose e frequência $(20 \mu \mathrm{g} / \mathrm{Kg} / \mathrm{BID})$ \\
$1,5-4,0$ & $>5,0$ & Diminuir dose $(<15 \mu \mathrm{g} / \mathrm{Kg} / \mathrm{SID})$
\end{tabular}

\section{RESULTADOS}

Nossa casuística foi composta de 30 cães com diagnóstico de hipotiroidismo primário adquirido, atendidos no serviço de Clínica Médica do Hospital Veterinário da Faculdade de Medicina Veterinária da Universidade Guarulhos (UnG) e no Serviço de Endocrinologia de duas clínicas particulares da cidade de São Paulo (Hospital Veterinário Pompéia e NAYA Especialidades) no período de 2009 a 2011.

Todos os cães avaliados tiveram sintomas clínicos compatíveis com a doença e valores baixos de T4 livre com média $0,21 \pm 0,14 \mathrm{ng} / \mathrm{dL}(\mathrm{VR}=0,7-2,6)$. A hipercolesterolemia e a hipertrigliceridemia foram identificadas em $66,6 \%$ $(\mathrm{n}=20 / 30)$ e $56,6 \%(\mathrm{n}=17 / 30)$ da nossa casuística, sendo a média e o desvio padrão de $434 \pm 315 \mathrm{mg} / \mathrm{dL}$ e $248 \pm 238 \mathrm{mg} /$ $\mathrm{dL}$, respectivamente. Os dados referentes à idade, sexo, raça e exames laboratoriais empregados para o diagnóstico 
Quadro 4. Resultados do teste pós-tiroxina, dose e frequência de administração da L-tiroxina e classificação dos animais de acordo com o peso corpóreo em grupos A ou B de 30 cães com hipotiroidismo primário (SP, 2009-11)

\begin{tabular}{|c|c|c|c|c|c|c|}
\hline Animal & Grupo A/B & Peso (kg) & $\begin{array}{l}\text { T4t basal } \\
(\mu \mathrm{g} / \mathrm{dL})\end{array}$ & $\begin{array}{c}\text { T4t 6h pós } \\
\text { tiroxina }(\mu \mathrm{g} / \mathrm{dL})\end{array}$ & $\begin{array}{l}\text { Dose da tiroxina } \\
(\mu \mathrm{g} / \mathrm{Kg})\end{array}$ & $\begin{array}{l}\text { Frequência de } \\
\text { administração }\end{array}$ \\
\hline 1 & B & 20,0 & 2,2 & 3,5 & 13 & SID \\
\hline 2 & B & 26,5 & 1,8 & 3,2 & 15 & BID \\
\hline 3 & A & 10,0 & 1,9 & 5,4 & 15 & BID \\
\hline 4 & B & 15,4 & 2,8 & 5,5 & 16 & BID \\
\hline 5 & A & 9,4 & 2,0 & 4,5 & 21 & BID \\
\hline 6 & B & 20,1 & 3,8 & 4,6 & 20 & BID \\
\hline 7 & B & 17,5 & 2,6 & 4,5 & 17 & SID \\
\hline 8 & B & 37,0 & 1,9 & 4,4 & 20 & SID \\
\hline 9 & B & 14,0 & 1,9 & 2,9 & 11 & SID \\
\hline 10 & A & 6,6 & 1,9 & 4,8 & 15 & SID \\
\hline 11 & A & 5,0 & 2,1 & 2,6 & 15 & BID \\
\hline 12 & B & 44,0 & 1,5 & 3,4 & 15 & SID \\
\hline 13 & A & 10,0 & 1,5 & 3,1 & 15 & SID \\
\hline 14 & B & 18,0 & 2,3 & 4,3 & 17 & SID \\
\hline 15 & A & 7,3 & 2,4 & 3,2 & 15 & BID \\
\hline 16 & B & 20,8 & 1,6 & 5,0 & 17 & SID \\
\hline 17 & B & 28,7 & 1,5 & 2,8 & 10 & SID \\
\hline 18 & A & 10,0 & 3,0 & 4,4 & 15 & SID \\
\hline 19 & A & 7,0 & 1,9 & 2,8 & 15 & SID \\
\hline 20 & A & 10,0 & 2,9 & 5,1 & 15 & SID \\
\hline 21 & B & 40,0 & 2,5 & 3,0 & 20 & BID \\
\hline 22 & B & 25,0 & 2,5 & 5,5 & 20 & BID \\
\hline 23 & B & 23,0 & 2,7 & 4,5 & 20 & BID \\
\hline 24 & B & 45,0 & 2,9 & 3,9 & 20 & BID \\
\hline 25 & A & 4,0 & 3,2 & 4,0 & 20 & BID \\
\hline 26 & B & 17,0 & 3,0 & 4,8 & 21 & BID \\
\hline 27 & B & 45,0 & 2,6 & 3,7 & 20 & BID \\
\hline 28 & A & 6,0 & 1,5 & 3,2 & 17 & SID \\
\hline 29 & A & 7,0 & 1,6 & 2,7 & 21 & BID \\
\hline 30 & B & 25,0 & 2,5 & 4,2 & 20 & SID \\
\hline Média & & 19,1 & 2,3 & 4,0 & 17 & \\
\hline \multicolumn{2}{|c|}{ Desvio padrão } & 12,6 & 0,59 & 0,96 & 3 & \\
\hline
\end{tabular}

Quadro 5. Valores médios de peso e dosagem de L-tiroxina empregada nos cães hipotiroideos dos grupos $A$ (peso $\leq 10$ kg) e $B$ (peso > $10 \mathrm{~kg})$, (SP, 2009-11)

\begin{tabular}{lccccc}
\hline & \multicolumn{2}{c}{ Grupo A (peso $\leq 10 \mathrm{~kg})$} & & \multicolumn{2}{c}{ Grupo B (peso > 10 kg) } \\
\cline { 2 - 3 } & Peso $(\mathrm{kg})$ & $\begin{array}{c}\text { Dose da tiroxina } \\
(\mu \mathrm{g} / \mathrm{Kg})\end{array}$ & & Peso $(\mathrm{kg})$ & $\begin{array}{c}\text { Dose da tiroxina } \\
(\mu \mathrm{Kg} / \mathrm{Kg})\end{array}$ \\
\hline Média & 7,7 & 16 & & 26,8 & 17 \\
Desvio padrão & 2,1 & 3 & & 10,7 & 3
\end{tabular}

de hipotiroidismo em 30 cães estão descritos no Quadro 1.

Os animais foram tratados com levotiroxina sódica humana (Synthroid ${ }^{\circledR}$, ABBOTT) por via oral, com a dose inicial de $10 \mu \mathrm{g} / \mathrm{kg} / \mathrm{SID}$ durante os primeiros sete dias e, posteriormente, $15 \mu \mathrm{g} / \mathrm{kg} / \mathrm{SID}$. Após 30 dias, os animais foram submetidos a um exame clínico e foi realizado o teste pós-tiroxina, exame este capaz de indicar se a posologia empregada está adequada ou se há necessidade de alterar a dose ou frequência de administração do fármaco. Valores de T4t basal de 1,5 a 4,0 e de T4t 6 horas pós-tiroxina de 2,5 a 5,0 foram considerados normais. Valores superiores ou inferiores a esses apontaram a necessidade de adequação da dose conforme o Quadro 3.

Dessa forma, a dose de $15 \mu \mathrm{g} / \mathrm{Kg}$ pode ser mantida em dez animais (33,3\% dos casos), dos quais $6(20 \%)$ a administração persistiu a cada 24 horas e quatro $(13,3 \%)$ passou a cada 12 horas. Em 17 animais $(56,6 \%)$ a dose precisou ser aumentada, em $3(10 \%)$ a dose precisou ser reduzida e $50 \%$ dos animais passaram a receber medicação a cada 12 horas. Com os novos ajustes na terapia, foi possível constatar a normalização dos valores de T4t no próximo teste pós-tiroxina realizado em 30 dias (Quadro 4).

A dose média de tiroxina utilizada em nossa casuística foi de $16,9 \pm 3,1 \mu \mathrm{g} / \mathrm{Kg}$ (Quadro 4), sendo a dose mínima de $10 \mu \mathrm{g} / \mathrm{kg}$ e a dose máxima de $21 \mu \mathrm{g} / \mathrm{kg}$. A dose final utilizada nos 30 cães hipotiroideos foi de 20 a $21 \mu \mathrm{g} / \mathrm{kg}$ em $40 \%$ dos animais ( $\mathrm{n}=12 / 30)$, de 15 a $19 \mu \mathrm{g} / \mathrm{kg}$ em $50 \%(\mathrm{n}=15 / 30)$ e de 10 a $14 \mu \mathrm{g} / \mathrm{kg} \mathrm{em} 10 \%$ dos casos (n=3/30)(Fig. 1). A dose

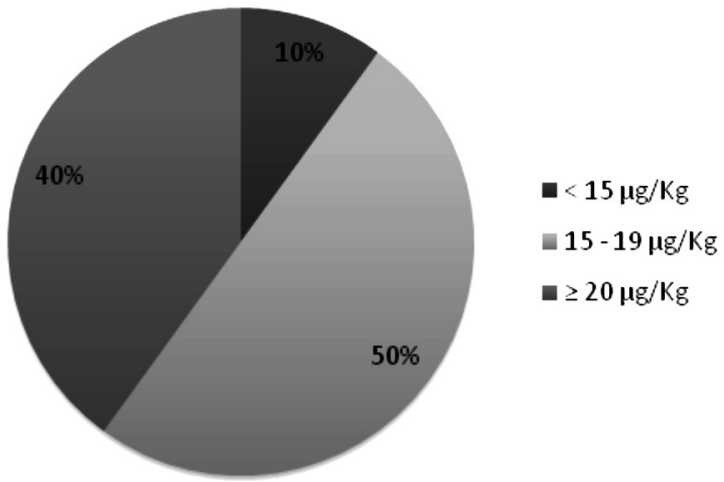

Fig.1. Dose de L-tiroxina $(\mu \mathrm{g} / \mathrm{kg})$ utilizada na terapia de 30 cães com hipotiroidismo primário adquirido. 
de 20 ou $21 \mu \mathrm{g} / \mathrm{kg}$ a cada 12 horas, a mais recomendada pela literatura, foi instituída em dez animais (33\% dos casos).

Diante de tamanha variação na dose de tiroxina empregada para se obter um controle clínico e hormonal satisfatório do hipotiroidismo, foi levantada a hipótese da dose variar de acordo com o peso do animal, uma vez que cães de pequeno porte apresentam maior taxa metabólica que cães de grande porte. Para tanto, os animais foram agrupados de acordo com o peso corpóreo em grupo A, cães com peso inferior ou igual a $10 \mathrm{~kg}(\mathrm{n}=12 / 30$; peso médio e desvio padrão de $7,7 \pm 2,1 \mathrm{~kg}$ ) e grupo $B$, cães com peso superior a 10 $\mathrm{kg}(\mathrm{n}=18 / 30,26,8 \pm 10,7 \mathrm{~kg})$. A dose média de tiroxina empregada nos grupos A e B foram, respectivamente, $16 \pm 3 \mu \mathrm{g}$ / $\mathrm{kg}$ e $17 \pm 3 \mu \mathrm{g} / \mathrm{kg}$ (Quadro 5). Tais valores não apresentaram diferença estatística (teste $t$ não pareado, $p=0,439$ ). Além disso, não houve correlação linear entre a dose de L-tiroxina utilizada e o peso dos animais (coeficiente de Pearson $r=0.2295$ ). A frequência de administração foi $50 \%$ a cada 24 horas (SID) e $50 \%$ a cada 12 horas (BID) para ambos os grupos (Fig.2 e 3).

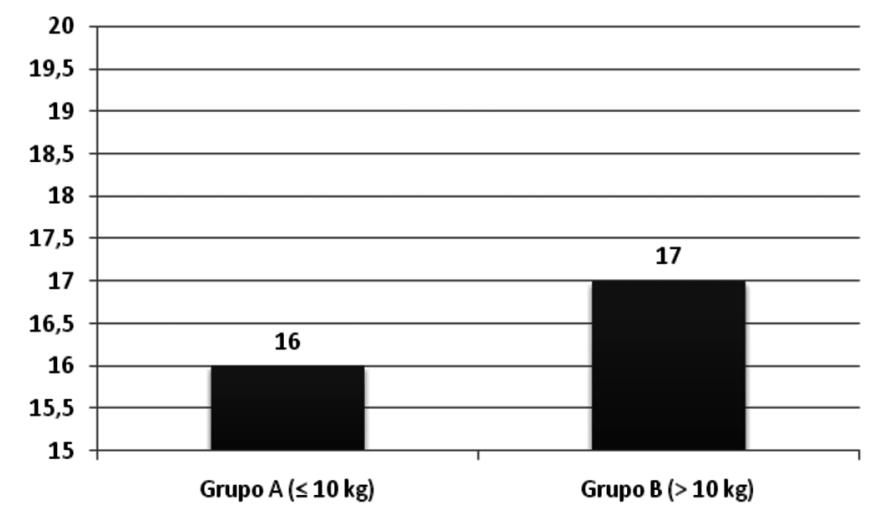

Fig.2. Dose média de L-tiroxina $(\mu \mathrm{g} / \mathrm{kg})$ utilizada na terapia de cães com hipotiroidismo primário adquirido do Grupo A $(\mathrm{n}=12$, peso $\leq 10 \mathrm{~kg})$ e do Grupo $\mathrm{B}(\mathrm{n}=18$, peso $>10 \mathrm{~kg})$

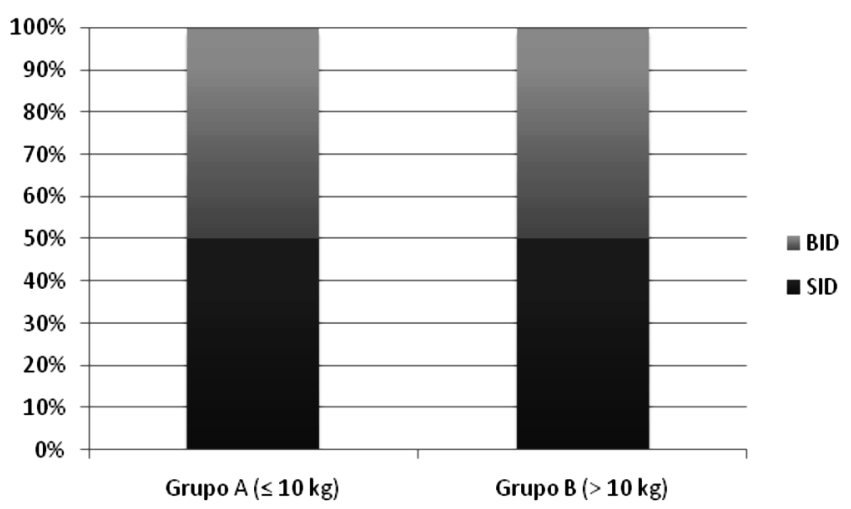

Fig.3. Frequência de administração (SID ou BID) de L-tiroxina na terapia de cães com hipotiroidismo primário adquirido do Grupo A $(n=12$, peso $\leq 10 \mathrm{~kg})$ e do Grupo B $(n=18$, peso $>10$ $\mathrm{kg}$ )

\section{DISCUSSÃO}

As características epidemiológicas e clínicas dos cães hipotiroideos incluídos no presente trabalho, tais como raça, idade, sexo, sinais clínicos e alterações ao exame físico es- tão de acordo a literatura veterinária (Dixon 2009, Scott-Moncrieff 2010), acometendo com maior frequência animais entre 4 e 8 anos de idade, uma discreta predisposição para fêmeas $(56,7 \%, n=17 / 30)$, destacando-se as raças Golden retriever, Cocker e Maltês, dentre outras. No grupo estudado todos os animais apresentavam pelo menos um dos sintomas clínicos compatíveis com hipotiroidismo tais como letargia, intolerância ao exercício, ganho de peso, termofilia e alterações cutâneas como alopecia, cauda de rato e piodermite recidivante (Panciera 2001, Dixon 2009).

0 diagnóstico de hipotiroidismo foi baseado na mensuração do T4 livre pelo método de diálise bifásica que apresenta uma ótima correlação com o método de diálise de equilíbrio, associado ou não ao T4 total e ao TSH de acordo com as condições financeiras dos proprietários. Quando a suspeita clínica do hipotiroidismo é grande e a síndrome do eutiroideo doente é descartada, a mensuração isolada do T4 livre tem um forte valor diagnóstico, pois é o teste que apresenta maior especificidade (94\%) e acurácia (95\%), quando avaliado isoladamente (Ramsey \& Herrtage 1997, Scott-Moncrieff 2010). A média de T4 livre observada em nossa casuística foi de $0,21 \pm 0,14 \mathrm{ng} / \mathrm{dL}(\mathrm{VR}=0,7-2,6)$. Nos achados laboratoriais, verificou-se que $66,6 \%$ dos animais apresentaram concentrações elevadas de colesterol, visto que a depleção do hormônio tireoidiano causa estase biliar, redução da excreção de colesterol e diminuição da metabolização da lipoproteína LDL, principal carreadora do colesterol. A frequência da hiperlipidemia encontrada foi semelhante àquela, geralmente, citada pela literatura de 75 a $80 \%$ (Catharine et al 2007, Dixon 2009), sendo que um dos animais apresentou níveis extremamente elevados de colesterol (1.850mg/dL).

No que se refere ao tratamento, todos os animais receberam dose inicial de tiroxina igual a $10 \mu \mathrm{g} / \mathrm{kg} / \mathrm{SID}$ durante uma semana e, posteriormente, $15 \mu \mathrm{g} / \mathrm{kg} / \mathrm{SID}$, dose essa que foi elevada para $20 \mu \mathrm{g} / \mathrm{kg}$, conforme a necessidade clínica e níveis sanguíneos de T4t dos animais. Esse aumento gradual da dose é indicado principalmente se o animal estiver debilitado ou se for cardiopata para que haja uma adaptação fisiológica do organismo aos níveis hormonais mais elevados. Dentre os 30 cães hipotiroideos estudados, 12 (40\%) foram tratados com dose entre 20 e $21 \mu \mathrm{g} / \mathrm{kg}, 15$ (50\%) com dose de 15 a $19 \mu \mathrm{g} / \mathrm{kg}$ e 3 cães (10\%) com dose de 10 a $14 \mu \mathrm{g} / \mathrm{kg}$. A dose máxima de 20 ou $21 \mu \mathrm{g} / \mathrm{kg}$ a cada 12 horas foi instituída em dez animais (33\% dos casos).

É importante notar ainda que, dos 10 cães tratados satisfatoriamente com $15 \mu \mathrm{g} / \mathrm{kg}$ (protocolo sugerido inicialmente), seis deles (60\%) não necessitaram de administração da L-tiroxina a cada 12 horas, pois apresentaram bom controle clínico e hormonal com uma única administração diária, mesmo sendo a tiroxina utilizada um produto humano.

Foi possível constatar que 50\% ( $n=15 / 30)$ dos animais apresentaram controle terapêutico adequado quando submetidos à terapia a cada 24 horas (SID), contrapondo-se à literatura veterinária que, usualmente, preconiza a administração de tiroxina a cada 12 horas (Feldman \& Nelson 2004, Scott-Moncrieff \& Guptill 2004). Recentemente, uma solução líquida de levotiroxina sódica veterinária 
(Levanta ${ }^{\circledR}$, IntervetInc) apresentou maior biodisponibilidade em comparação aos tabletes de L-tiroxina veterinária, se mostrando eficaz na maioria dos cães hipotiroideos com uma única administração diária (Traon et al. 2010).

Essas diferenças de dose e frequência de administração da L-tiroxina na suplementação hormonal do hipotiroideo justificam-se pelas variações individuais de absorção e metabolismo do fármaco (Feldman \& Nelson 2004). 0 pico da droga na circulação sanguínea pode variar bastante entre os cães devido a diferenças na sua meia vida plasmática, estimada em torno de 10 a 14 horas. A terapia a cada 24 horas beneficia cães com baixo metabolismo e duração mais prolongada do efeito do fármaco no organismo. Nesse caso, se o animal for submetido a uma terapia a cada 12 horas e na dose máxima, o risco de tireotoxicose aumenta (Richard 1989). Nesse contexto, poderíamos esperar que cães de grande porte se beneficiariam mais com administração SID de L-tiroxina e cães de pequeno porte com administração BID. No entanto, ao analisarmos as diferentes faixas de peso corpóreo, não foi observada qualquer diferença na dose de tiroxina empregada $(16 \pm 3 \mu \mathrm{g} / \mathrm{kg}$ e $17 \pm 3 \mu \mathrm{g} / \mathrm{kg})$ nos grupos A (cães com peso $\leq 10 \mathrm{~kg}$ )e B (cães com peso $>10$ $\mathrm{kg}$ ), respectivamente, bem como na frequência de administração (50\% SID e 50\% BID nos dois grupos).

Embora a utilização de doses fracionadas certamente resulte em menor flutuação das concentrações de T4 circulante e menor risco de concentrações suprafisiológicas no pico de ação do fármaco em comparação à administração da mesma dose diária in bolus (Nachreiner 1993), a ação biológica dos hormônios tiroideanos pode ultrapassar a ação da meia vida plasmática, explicando o sucesso do tratamento uma vez ao dia em muitos casos (Richard 1989, De Marco 2008).

A tireotoxicose, embora rara, pode se desenvolver em cães tratados com doses excessivas de L-tiroxina, especialmente naqueles medicados a cada 12 horas, sendo fundamental a monitorização frequente da terapia com testes hormonais, notadamente o teste pós-tiroxina. Os sintomas clínicos da tireotoxicose caracterizam-se por: poliúria, polidipsia, polifagia, perda de peso, hiperatividade, taquicardia, vômito e diarréia (Hansen et al. 1992). Nessas condições, o hormônio tiroideano deve ser interrompido até melhora clínica e, posteriormente, uma redução de 25 a $50 \%$ na dose atual ou alteração na frequência de administração da droga deve ser instituída (Feldman \& Nelson 2004).

Foi observado no presente trabalho uma grande variação nos protocolos terapêuticos de cães hipotiroideos, com doses de L-tiroxina variando de 10 a $21 \mu \mathrm{g} / \mathrm{kg}$, além de administração a cada 24 horas em $50 \%$ dos casos. A variabilidade de absorção e metabolismo da L-tiroxina não parece se correlacionar com o peso do animal, sendo imprevisível quem deverá receber dose e frequência máxima da medicação. Desta forma, o protocolo deve ser individualizado e o paciente devidamente monitorado. Sugere-se que a terapia se inicie com a dose de $15 \mu \mathrm{g} / \mathrm{kg} / \mathrm{SID}$ e, após a realização do teste pós tiroxina, a dose e frequência da L-tiroxina sejam ajustadas de acordo com a necessidade do animal.

\section{REFERÊNCIAS}

Catharine S.M. 2007. Clinical signs and concurrent diseases of hipothyroidism in dogs and cats. Vet. Clin. Small Anim. Pract. 37(4):709-722.

Daminet S. \& Ferguson D.C. 2003. Influence of drugs on thyroid function in dogs. J. Vet. Intern. Med. 17:463-72.

De Marco V. 2008. Terapêutica das principais endocrinopatias em cães e gatos, p.423-427. In: Andrade S. (Ed.), Manual de Terapêutica Veterinária. 3a ed. Roca, São Paulo.

Dixon R.M., Reid J.W.S. \& Money T.C. 2002. Treatment and therapeutic monitoring of canine hypothyroidism. J. Small Anim. Pract. 43(8):334-340.

Dixon R.M. 2009. Hipotireoidismo canino, p.911-14.In: Mooney C.T. \&Peterson M.E. (Eds), Manual de Endocrinologia Canina e Felina. $3^{\mathbf{a}}$ ed. Roca, São Paulo.

Feldman E.C \& Nelson R.W. 2004. Hipotiroidismo, p.86-142. In: Feldman E. C. \& Nelson R.W. (Eds), Canine and Feline Endocrinology and Reproduction. $3^{\text {rd }}$ ed. W.B. Saunders, Philadelphia.

Graham A.P., Refsal R.K. \& Nachreiner F.R. 2007. Etiopathologic findings of canine hypothyroidism. Vet. Clin. North Am. Small Anim. Pract. 37(4):618-661.

Hansen S.R., Timmons S.P. \& Dorman D.C. 1992. Acute overdose of levothyroxine in a dog. Am. J. Vet. Med. Assoc. 200(10):1512-1514.

Nachreiner R.F., Refsal K.R., Ravis W.R., Hauptman J., Rosser E.J. \& Pedersoll W.M. 1993. Pharmacokinetics of L-thyroxine after its oral administration in dogs. Am. J. Vet. Res. 54(12): 2091-2098.

Nelson R.W. \& Elliott D. 2006. Distúrbios endócrinos, p.665-682. In: Nelson R.W. \& Couto G.C. (Eds), Medicina Interna de pequenos animais. $3^{\mathbf{a}}$ ed. Elsevier, Rio de Janeiro.

Nelson R.W. 1989. Treatment of canine hypothyroidism, p.993-1000. In: Bonagura J.D. (Ed.), Kirk's Current Veterinary Therapy x Small Animal Practice. $10^{\text {th }}$ ed. W.B. Saunders, Philadelphia, Pennsylvania.

Panciera D.L. 2001. Conditions associated with canine hypothyroidism. Vet. Clin. North Am., Small

Peterson M.E., Melian C. \& Nichols R. 1997. Measurement of serum total thyroxine, triiodothyronine, free thyroxine and thyrotropin concentrations for diagnosis of hypothyroidism in gos. J. Am. Vet. Med. Assoc. 211(11):1396-1401.

Ramsey I.K. \& Herrtage M.E. 1997. Thyroid stimulating hormone and total thyroxine concentrations in euthyroid, sick euthyroid and hypothyroid dogs. J. Small Anim. Pract. 38:540-545.

Scott-Moncrieff J.C.R. 2010. Hypothyroidism, p.160-175. In: Ettinger S.J. \& Feldman E.C. (Eds), Textbook of Veterinary Internal Medicine. $7^{\text {th }}$ ed. Elsevier, Rio de Janeiro.

Traon G.L., Brennan S.F., Burgaud S., Daminet S., Gommeren K., Horspool L.J.I., Rosenberg D. \& Mooney C.T. 2009. Clinical evaluation of a novel liquid formulation of L-thyroxine for once daily treatment of dogs with hypothyroidism. J. Vet. Intern. Med. 23(1):43-49. 